\title{
Electron-phonon renormalization of the absorption edge of the cuprous halides
}

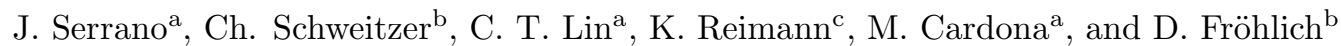 \\ ${ }^{a}$ Max-Planck-Institut für Festkörperforschung, Heisenbergstr. 1, 70569 Stuttgart, Germany \\ ${ }^{\mathrm{b}}$ Institut für Physik, Universität Dortmund, 44221 Dortmund, Germany \\ ${ }^{\mathrm{c}}$ Max-Born-Institut für Nichtlineare Optik und Kurzzeitspektroskopie, 12489 Berlin, Germany
}

(October 31, 2018)

\begin{abstract}
Compared to most tetrahedral semiconductors, the temperature dependence of the absorption edges of the cuprous halides $(\mathrm{CuCl}, \mathrm{CuBr}, \mathrm{CuI})$ is very small. $\mathrm{CuCl}$ and $\mathrm{CuBr}$ show a small increase of the gap $E_{0}$ with increasing temperature, with a change in the slope of $E_{0}$ vs. $T$ at around $150 \mathrm{~K}$ : above this temperature, the variation of $E_{0}$ with $T$ becomes even smaller. This unusual behavior has been clarified for $\mathrm{CuCl}$ by measurements of the low temperature gap vs. the isotopic masses of both constituents, yielding an anomalous negative shift with increasing copper mass. Here we report the isotope effects of $\mathrm{Cu}$ and $\mathrm{Br}$ on the gap of $\mathrm{CuBr}$, and that of $\mathrm{Cu}$ on the gap of $\mathrm{CuI}$. The measured isotope effects allow us to understand the corresponding temperature dependences, which we also report, to our knowledge for the first time, in the case of CuI. These results enable us to develop a more quantitative understanding of the phenomena mentioned for the three halides, and to interpret other anomalies reported for the temperature dependence of the absorption gap in copper and silver chalcogenides; similarities to the behavior observed for the copper chalcopyrites are also pointed out.
\end{abstract}

PACS: 71.35.Cc,71.36.+c,63.20.Kr

\section{INTRODUCTION}

The electronic band structure of the cuprous halides differs considerably from that of other tetrahedral semiconductors which contain neither copper nor silver as a cation. It was early recognized that the differences are due to a strong admixture of the $3 d^{10}$ copper electrons ( $4 d^{10}$ in the case of silver) to the conventional, valence-band-forming $p$ electrons of the anion $\left(2 p^{7}\right.$ for chlorine). Instead of the conventional 8 valence electrons of the primitive cell of zincblende $\left(s^{2}+p^{6}\right)$ a total of 18 $\left(d^{10}+s^{2}+p^{6}\right)$ hybridized electron states constitute the valence bands of the cuprous halides. The ten $d$ electron states split in the cubic field into six $\Gamma_{15}$ and four $\Gamma_{12}$ states. The former hybridize strongly with the six $p$-like valence states of the halogen atoms, whereas the $\Gamma_{12}$ states do not hybridize at $\mathbf{k}=0$, but they hybridize with $s$ and $p$ states at a general point of the Brillouin zone (BZ) (continuity requires this hybridization to remain small). This hybridization leads to a number of anomalies, several of them have been discovered after the original suggestion in Ref. 1 (for an early review see Ref. 22). Among them, we first mention that the lowest gap of these materials (which is direct and occurs at the $\Gamma$ point, as in GaAs and $\mathrm{ZnSe}$ ) is lower than expected from extrapolation of their isoelectronic group IV, III-V and II-VI counterparts (Ge: $0.9 \mathrm{eV}$, GaAs: $1.5 \mathrm{eV}$, ZnSe: $2.7 \mathrm{eV}, \mathrm{CuBr}: 3.0 \mathrm{eV}$ ). Even more striking is the fact that the spin-orbit splitting $\Delta_{0}$ of the uppermost valence band $\left(\Gamma_{15}\right.$ splits into $\Gamma_{8}$ and $\Gamma_{7}$ double group representations is rather small, even negative $\left(\Gamma_{7}\right.$ above $\left.\Gamma_{8}\right)$ for $\mathrm{CuCl}$. 3 Additionally, anomalously small temperature and pressuret 6 dependences of the gap have also been observed.
The temperature dependence of semiconductor gaps is intrinsically connected to their dependence on isotopic mass. The reason for this is that the temperature dependence is caused, to a large part, by the renormalization of the band gap due to electron-phonon interaction. Because of the zero-point vibrations there is already an effect at zero temperature, which is proportional to the square of the vibrational amplitudes, obviously massdependent.

During the past ten years a number of experiments 12 has been performed on the dependence of the gap on isotopic mass. In compound semiconductors, such experiments yield considerably more information than temperature-dependent experiments, since the mass of each constitment element can have a different effect on a given gap. 612 Of particular interest is the case of $\mathrm{CuCl}$ : an increase of the chlorine mass (from ${ }^{35} \mathrm{Cl}$ to ${ }^{37} \mathrm{Cl}$ ) leads to the usual increase of the $E_{0}$ gap, whereas the increase of the copper mass (from ${ }^{63} \mathrm{C}$ t to ${ }^{65} \mathrm{Cu}$ ) results in an anomalous decrease of the gap. This led to the understanding of the anomalous temperature dependence of the $E_{0}$ gap of $\mathrm{CuCl}$ reported in Refs. 13 16. Using the same model and the observed data for $E_{0}(T)$ in $\mathrm{CuBr}$, the authors of Ref. 7 predicted the dependences of the $E_{0}$ gap on copper $\left(M_{\mathrm{Cu}}\right)$ and on bromine mass $\left(M_{\mathrm{Br}}\right)$.

In this paper we report measurements of $\partial E_{0} / \partial M_{\mathrm{Cu}}$ and $\partial E_{0} / \partial M_{\mathrm{Br}}$ for $\mathrm{CuBr}$, which confirm the above conjecture. We also report measurements of $\partial E_{0} / \partial M_{\mathrm{Cu}}$ for the $E_{0}$ gap of $\mathrm{CuI}$ (since iodine is one of the few elements with only one stable isotope, ${ }^{127} \mathrm{I}, \partial E_{0} / \partial M_{\mathrm{I}}$ could not be measured). For $\mathrm{CuI}$ we also present rather unusual results on the temperature dependence of the $E_{0}$ gap. In spite of the missing value of $\partial E_{0} / \partial M_{\text {I }}$ for $\mathrm{CuI}$, still a detailed analysis of electron-phonon renormalization effects 
is possible. This is due to the weak and considerably structured temperature dependence, as reported in this work. In the temperature range from 0 to $300 \mathrm{~K}$ the $E_{0}$ gap decreases by $8 \mathrm{meV}$, in agreement with the early data of Ref. 1. A fit to the experimental $E_{0}(T)$ data with a three-oscillator model yields values for $\partial E_{0} / \partial M_{\mathrm{I}}$ and for $\partial E_{0} / \partial M_{\mathrm{Cu}}$, the latter value in good agreement with the directly measured one. The results we obtained for the cuprous halides are used to interpret the anomalies reported for the temperature dependence of the lowest band gap in other tetrahedral compounds like cuprous and silver chalcopyrites 1724 which exhibit a small positive slope at low temperatures followed by a sign reversal in the slope above $\approx 100 \mathrm{~K}$.

This article is organized as follows: We discuss in the next section the peculiar band structure of the cuprous halides in the region around the $E_{0}$ gap. Section III contains details about the experimental method. In Sec. IV we present our data for the isotopic dependence of $E_{0}$ at low temperature for $\mathrm{CuI}$ and $\mathrm{CuBr}$ and for the temperature dependence of $E_{0}$ for CuI. Then we introduce in Sec. $\mathrm{V}$ a model for both temperature and isotope effects and compare our present results with those obtained in Ref. 7 for $\mathrm{CuCl}$. Finally, in Sec. VI, an exhaustive compilation of the anomalies reported in the behavior of the temperature dependence of $E_{0}$ is performed for the cuprous and silver chalcopyrites and quantitatively interpreted in terms of our results for the simpler cuprous halides.

\section{ELECTRONIC BAND STRUCTURE}

The lowest absorption edge (equivalently referred to as lowest gap or lowest exciton) of $\mathrm{CuCl}$ differs considerably from that of $\mathrm{CuBr}$ and CuI. The difference is due to the anomalous sign of the spin-orbit splitting, which is negatife for $\mathrm{CuCl}$, but positive for the other cuprous halides, 130 a consequence of the negative contribution of the copper $3 d$ electrons to the spin-orbit splitting. 3 Hence the symmetry of the uppermost valence band of $\mathrm{CuBr}$ and $\mathrm{CuI}\left(E_{0}\right)$ is $\Gamma_{8}$, whereas that of $\mathrm{CuCl}$ is $\Gamma_{7}$ $\left(E_{0}+\Delta_{0}\right)$. The symmetry of the lowest conduction band is $\Gamma_{6}$ in all cases.

Because of Coulomb interaction, electrons in the conduction band and holes in the valence band form exciton states. Excitons corresponding to the $\Gamma_{8}$ valence band are often labeled $Z_{1,2}$, where $Z$ stands for zincblende, whereas excitons belonging to the $\Gamma_{7}$ valence band are labeled $Z_{3}$. The exciton symmetry is given by the direct product of conduction and valence band symmetries. Thus $Z_{1,2}$ excitons $\left(\Gamma_{8} \times \Gamma_{6}\right)$ are eightfold degenerate and split through exchange interaction into $\Gamma_{3}, \Gamma_{4}$, and $\Gamma_{5}$ components 25 whereas the $Z_{3}$ fourfold degenerate excitons $\left(\Gamma_{7} \times \Gamma_{6}\right)$, split into $\Gamma_{2}$ and $\Gamma_{5}$. Only the $\Gamma_{5}$ components are dipole allowed and contribute to one-photon absorption processes. Two-photon transitions, on the other hand, are allowed for $\Gamma_{1}, \Gamma_{3}, \Gamma_{4}$ and $\Gamma_{5}$ excitons. Each of the $\Gamma_{5}$ excitons constitutes a triplet, whose transverse components interact strongly with photons, thus giving rise to exciton-photon coupled states (polaritons).

\section{EXPERIMENT}

We have performed measurements on samples of $\mathrm{CuI}$ and $\mathrm{CuBr}$ with all possible combinations of stable isotopes $\left({ }^{63} \mathrm{Cu},{ }^{65} \mathrm{Cu} ;{ }^{127} \mathrm{I} ;{ }^{79} \mathrm{Br},{ }^{81} \mathrm{Br}\right)$ and the corresponding natural compositions. Details gf the crystal growth have been published elsewhere.26 28

To be able to accurately determine the rather small isotope shifts, we have used the nonlinear optical technique of two-photon absorption 29 (TPA), since this technique yields much smaller line widths than linear optical methods, as, e.g., reflection or absorption (as an example, for $\mathrm{CuCl}$ one can compare the absorption data in Ref. 1 with the two-photon data in Refs. 7 and 30). For the same reason we have restricted our measurements to the excitons with the lowest energies, i. e., to the $Z_{1,2}$ excitons in $\mathrm{CuBr}$ and $\mathrm{CuI}$, since excitons with higher energies are broadened through phonon-induced decay into lower-energy states.

The classical method 30 for the measurement of TPA is to detect a change in the intensity of the transmitted light of the first beam during the presence of the second (highintensity) beam. This method is, however, not sensitive enough to detect TPA in our thin samples. Therefore in our case TPA is detected by monitoring the luminescence intensity from a defect-related level below the band gap as a function of the sum of the two photon energies (the two-photon energy). Since we use two photons from the same laser, the two-photon energy is equal to twice the laser photon energy.

The experimental setup for these measurements consists of an exciting laser, a cryostat with the sample and the detection system.

Since non-resonant in the intermediate state (the photon energies are far from resonances in our experiments) two-photon absorption in these compounds is a very weak effect; high excitation intensities of about $10 \mathrm{MW} / \mathrm{cm}^{2}$ must be used. They are generated by a Nd:YAG-laserpumped tunable dye (styril) laser with a pulse width of $5 \mathrm{~ns}$ and a repetition rate of $10 \mathrm{~Hz}$.

The samples were mounted strain-free in a helium flow cryostat. The temperature, which is measured with a calibrated silicon diode mounted near the samples, can be varied by changing the rate of helium flow and by additional heating. For the measurements of the isotopic effect on the band gap the samples were held at a temperature of $7 \mathrm{~K}$.

The detection system consists of a $f / 1.8$ collection optics, a prism spectrometer and appropriate optical filters to separate the weak luminescence from the intense laser light, and a bialkali photomultiplier. The wavelength of 
the spectrometer is held fixed at the luminescence maximum. The output signal from the photomultiplier is fed into a gated integrator and sent via an analog-digital converter to a personal computer, which also controls the scanning of the dye laser.

\section{RESULTS}

\section{A. CuI}

Two-photon absorption spectra for $\mathrm{CuI}$ with different copper isotopic compositions are shown in Fig. 1. The lower and higher energy peaks we observe are identified as the $\Gamma_{3}$ (with possible admixture of $\Gamma_{5}$ transverse) and $\Gamma_{5}$ longitudinal excitons 25,31 The splitting of $6.7 \mathrm{meV}$ between the peaks is in agreement with previously reported values31 (6.5 meV). Shown in Fig. 28 are the peak energies obtained from a Lorentzian fit vs. the average isotopic mass. The values of $\partial E_{0} / \partial M_{\mathrm{Cu}}$ from a linear leastsquares fit are listed in Table Although the error bars of the $\Gamma_{3}$ data, $-550 \pm 12 \mu \mathrm{eV} / \mathrm{amu}$, are much smaller than those of the weaker $\Gamma_{5}$ data, $-510 \pm 150 \mu \mathrm{eV} / \mathrm{amu}$, the agreement between both sets of data is excellent.

Figure 3 shows the measured temperature dependence of the lower-frequency peak of Fig. 11 for natural $\mathrm{CuI}$ for temperatures up to $300 \mathrm{~K}$. Measurements at higher temperature are not meaningful because of the increasing line width (see error bars in Fig. 3). One should note the following important points:

1. The overall effect of temperature is a decrease in energy, opposite to the observations for $\mathrm{CuCl}$ and $\mathrm{CuBr}$.

2. At about $150 \mathrm{~K}$ a change in the slope of $E_{0}(T)$ is seen, remindful of the behavior observed for $\mathrm{CuCl}$ and $\mathrm{CuBr}$.

3. Below $60 \mathrm{~K}$ a weak sigmoidal behavior is observed, with maxima at $T=0$ and $T=40 \mathrm{~K}$ (see inset in Fig. 3).

\section{B. $\mathrm{CuBr}$}

Some two-photon spectra for $\mathrm{CuBr}$ samples of different isotopic compositions measured at $7 \mathrm{~K}$ are displayed in Fig. 4. In contrast to $\mathrm{CuI}$ here one observes only the $\Gamma_{5}$ longitudinal exciton. Attempts to observe the $\Gamma_{3}$ exciton revealed that it is at least two orders of magnitude weaker than in $\mathrm{CuI}$. This may be related to the near degeneracy of the $\Gamma_{3}$ and $\Gamma_{5}$ excitons in $\mathrm{CuI}$, which does not occur 3132 in $\mathrm{CuBr}$. By means of Lorentzian fits we obtain the $E_{0}$ energies plotted in Fig. 5 as a function of the corresponding isotopic masses of copper and bromine. Least-squares fits to these points result in the corresponding slopes listed in Table The data allow us to establish the anomalous negative sign of the copper mass derivative which was predicted in Ref. 7 .

\section{DISCUSSION}

We assume here, as it was done in previousl 1 works, that shifts with either temperature or isotopic mass observed for the $Z_{1,2}$ excitons (or for $Z_{3}$ in the case of $\mathrm{CuCl}$ ) are representative of the corresponding one-electron gaps. This assumption is usually justified by the fact that the exciton binding energies, which are very large in the copper halides, are nevertheless more than one order of magnitude smaller than the corresponding band gap. Additionally, one has to consider that for $\mathrm{CuCl}$ (and to lesser extent also for $\mathrm{CuBr}$ ) the exciton binding energy 33 ( $\approx 190 \mathrm{meV}$ for $\mathrm{CuCl}, \approx 140 \mathrm{meV}$ for $\mathrm{CuBr}$ ) is close to or larger than another important characteristic energy, that of the spin-orbit splitting $\Delta_{0}(\approx-70 \mathrm{meV}$ for $\mathrm{CuCl}$, $\approx 150 \mathrm{meV}$ for $\mathrm{CuBr}$ ). Consequently the spinorbit splitting measured for the exciton should be somewhat smaller than the splitting $\Delta_{0}$ of the valence bands at the $\Gamma$ point of the BZ. This decrease in $\Delta_{0}$, which has been investigated for diamond, 34,35 has not been discussed for $\mathrm{CuCl}$ and $\mathrm{CuBr}$. It is nevertheless unlikely that changes in $\Delta_{0}$ induced by changes either of temperature or of mass will alter our conclusions. This has been confirmed for the temperature dependence 16 of $E_{0}$ and $E_{0}+\Delta_{0}$ in $\mathrm{CuBr}$ (see Fig. 8 of Ref. 7). In any case, for $\mathrm{CuI}$ the problem does not arise since $\Delta_{0} \approx 930 \mathrm{meV}$ is much larger than the exciton binding energy $36(\approx 58 \mathrm{meV})$.

The model we use here for the description of the mass and temperature dependences of the band gap is the same as in Ref. 6. The effects of both temperature and mass changes can be divided into the effect of the change of lattice constant and the renormalization by the electronphonon interaction. The change in lattice constant leads to the following change of the gap:

$$
\Delta_{\mathrm{th}} E_{0}(X)=3 \alpha \frac{a(X)-a_{0}}{a_{0}},
$$

with $\alpha=d E_{0} / d \ln V$ the deformation potential of the $E_{0}$ gap and $a(X)$ the lattice constant as a function of $X$ (temperature or one of the isotopic masses).

In principle, for the calculation of the renormalization by electron-phonon interaction it is necessary to include every possible phonon mode. It turns out, however, that a much simplified model is sufficient for a good description of the data. Instead of using the complete phonon dispersion, we use only two (three for $\mathrm{CuI}$ ) average phonons. This leads to the following expression:

$$
\Delta E(T)=\sum_{i} \frac{A_{i}}{\Omega_{i} M_{i}}\left[2 n_{B}\left(\frac{\Omega_{i}}{T}\right)+1\right] .
$$

The sum is over the oscillators used, $A_{i}$ is the electronphonon coupling coefficient for the oscillator $i, \Omega_{i}$ its 
frequency (in Kelvin), and $n_{B}$ the corresponding BoseEinstein factor. $M_{i}$ is the reduced mass for the vibration considered.

Two oscillators with opposite values of $A$ are required to describe the change in $E_{0}(T)$ observed at $\approx 100 \mathrm{~K}$ for both $\mathrm{CuCl}$ and $\mathrm{CuBr}$. The anomalous positive slope for $T<100 \mathrm{~K}$ is due to the copper vibrations $\left(A_{\mathrm{Cu}}>0\right)$, whereas the decrease in slope for $T>100 \mathrm{~K}$ is due to the vibrations of the halogen, which have negative values of A.

Since it is not clear a priori which phonons should be used, we compare in Table $\$$ different possibilities. We show in this table the experimental results for the isotope dependence together with theoretical values obtained from a two-oscillator fit of the temperature dependence. The three values given in Table 1 for $\mathrm{CuCl}$ and $\mathrm{CuBr}$ are obtained by choosing for the two oscillators (T) the average TO and the average TA phonon energy, (L) the LO and the LA phonon energy, or $(\mathrm{M})$ the average of all acoustic phonon energies and the average of all optical phonon energies, respectively. As one can see, the best agreement between measured and calculated values is reached for $\mathrm{CuCl}$ for case $\mathrm{T}$ (the same energies were also used in Ref. (7) and for $\mathrm{CuBr}$ for case M. At this point we should note that in Ref. 7 copper, in spite of its slightly lighter mass, seemed to play the main role in the acoustic vibrations, whereas bromine was responsible for the optical modes. This ad hoc assumption has recently been validated by Raman measurementse on isotopically tailored $\mathrm{CuBr}$. A similar assumption is also necessary to explain the present measurements.

In order to get an appropriate description of the temperature dependence of the gap of CuI shown in Fig. 3 . the use of a three-oscillator model is necessary. For the frequencies we choose the average TA, LA, and LO phonon energies. The results of this fit, together with the effect of thermal expansion, are shown by the dashed line in Fig. 3. Apart from an overestimate of the amplitude of the low-temperature wiggle, the fit agrees well with the experimental data. Using the model, we obtain a copper isotopic shift of $-524 \mu \mathrm{eV} / \mathrm{amu}$, which agrees perfectly with the measured values $(-550$ and $-510 \mu \mathrm{eV} / \mathrm{amu})$.

One should note that in $\mathrm{G}_{\mathrm{l}} \mathrm{I}$ the contribution to $E_{0}(T)$ due to thermal expansion, 37 which is shown by the diamonds in Fig. 3, is very important37 (it accounts for about two thirds of the total band gap change). This is in marked contrast to $\mathrm{CuCl}$ and $\mathrm{CuBr}$, for which the effect of the thermal expansion is negligible.

Figure 3 indicates that the net effect of the electronphonon interaction (dotted line in Fig. 3) is very small $(\leq 5 \mathrm{meV})$. This suggests that there is near total compensation between the effects of the copper and the iodine vibrations. The residual effects contain detailed structure, which requires three oscillators for its description. We have placed these three oscillators at the average TA frequency $(60 \mathrm{~K})$, LA frequency $(156 \mathrm{~K})$ and LO frequency $(224 \mathrm{~K})$. An additional oscillator (e. g., at the TO frequency) does not improve the quality of the fit. As adjustable parameters we use the corresponding three coefficients $A_{i} /\left(\Omega_{i} M_{i}\right)$ of Eq. (2). In this manner we obtain, after adding the thermal expansion effect, the dashed line of Fig. 3. The coefficient $A_{i} / \Omega_{i} M_{i}$ that corresponds to the TA phonons is found to be about one tenth of that of the other two phonons. Because of the heavier mass of iodine, the LA coefficient should be proportional to $\partial E_{0} / \partial M_{\mathrm{I}}$, according to Eq. 2, whereas the LO coefficient should correspond to $\partial E_{0} / \partial M_{\mathrm{Cu}}$, neglecting the TA coefficient. The corresponding values of $\partial E_{0} / \partial M_{\mathrm{Cu}}$ and $\partial E_{0} / \partial M_{\mathrm{I}}$ are listed in Table 1 . The fitted value of $\partial E_{0} / \partial M_{\mathrm{Cu}}=-524 \pm 16 \mu \mathrm{eV} / \mathrm{amu}$ (the error bars have been estimated from the value of the TA coefficient, which was neglected when determining $\left.\partial E_{0} / \partial M_{\mathrm{Cu}}\right)$ agrees very well with the directly measured one $(-550 \pm 12 \mu \mathrm{eV} / \mathrm{amu})$.

\section{CHALCOPYRITES}

Information about the temperature dependence of the lowest gaps (usually direct and also labeled $E_{0}$ ) of the copper and silver chalcopyrites (I-III- $\mathrm{VI}_{2}$ compounds) is found scattered throughout the literature, mostly limited to the $0-300 \mathrm{~K}$ region. 1724 Most of these data show anomalies similar to those observed for the cuprous halides, a fact which seems to have remained unnoticed by most authors (note, however, that in Ref. 18 ad hoc fits with two oscillators, with contributions of opposite signs, were performed). We have listed in Table II the gaps of a number of such chalcopyrites and the change they experience from $0 \mathrm{~K}$ to $300 \mathrm{~K}$, together with the temperature $T_{a}$ at which a change in the slope of $E_{0}(T)$ is observed. We have also included in the last column the corresponding deformation potentials $\alpha=d E_{0} / d \ln V$, taken from Refs. 19 and 33. For comparison we have also shown the available corresponding values for the cuprous halides, for wurtzite structure $\mathrm{AgI}(\mathrm{AgCl}$ and $\mathrm{AgBr}$ crystallize in the rocksalt structure, therefore presenting a very different behavior), and for several zincblende-type III-V and II-VI compounds. The trends observed for these parameters in the chalcopyrites reinforce the arguments made in this article concerning the anomalous sign of the copper (which also applies to silver) contributions to the properties under discussion, to which we can add now the deformation potential $\alpha$ :

1. The differences $E_{0}(300 K)-E_{0}(0)$ reverse sign and become smaller in magnitude when going from the III-V and II-VI compounds to $\mathrm{CuCl}$ and $\mathrm{CuBr}$.

2. For $\mathrm{CuI}$ and $\mathrm{AgI}$ these differences also become very small although the sign reversal does not quite take place.

3. The corresponding behavior for the chalcopyrites lies between that of the II-VI and the I-VII compounds. 
This last statement is reinforced when following the isoelectronic series:

$\begin{array}{lcccc} & \text { GaAs } & \text { ZnSe } & \mathrm{CuGaSe}_{2} & \mathrm{CuBr} \\ E_{0}(T=0)(\mathrm{meV}): & 1540 & 2770 & 1720 & 2967 \\ E_{0}(300 K)-E_{0}(0): & -95 & -90 & -35 & +32 \\ \alpha(\mathrm{eV}): & -9.8 & -4.9 & -3.9 & -0.3\end{array}$

The near cancellation of $E_{0}(300 K)-E_{0}(0)$ for $\mathrm{CuI}$ and its negative sign can also be followed through an isoelectronic series:

$\begin{array}{lcccc} & \text { GaSb } & \text { ZnTe } \mathrm{CuGaTe}_{2} & \mathrm{CuI} \\ E_{0}(T=0)(\mathrm{meV}): & 813 & 2381 & 1000 & 3057 \\ E_{0}(300 K)-E_{0}(0): & -88 & -111 & -43 & -8 \\ \alpha(\mathrm{eV}): & -7.0 & -5.9 & -3.4 & -1.1\end{array}$

Note that whereas in the GaAs series $E_{0}$ increases with temperature for the chalcopyrite at low temperature and decreases above $T_{a}$, for the GaSb series no increase is observed at low temperature, a fact which corresponds to the results of Fig. 3 for $\mathrm{CuI}$. The values of $E_{0}(300 \mathrm{~K})-E_{0}(0)$ for the chalcopyrites lie between those of the corresponding isoelectronic I-VII and II-VI compounds; this reflects the fact that only half as much copper (or silver) is present in the chalcopyrites as in the I-VII counterparts. Similar trends to those just described also apply to the deformation potential $\alpha$. Hence an anomalous, positive contribution to $\alpha$ must result from the presence of the noble metal.

We notice that most elements in the chalcopyrites of Table II have more than one stable isotope (exceptions: $\mathrm{Al}$, As and I). It would be most interesting to measure the corresponding isotope effects on the $E_{0}$ gaps of chalcopyrite samples.

\section{CONCLUSIONS}

We have measured the effect of changing copper isotopes on the two-photon spectra of $\mathrm{CuI}$ and the temperature dependence of the lowest exciton, corresponding to the $E_{0}$ gap. For $\mathrm{CuBr}$ we have measured the corresponding isotope effects for both constituents. The results have been used to interpret the anomalous temperature dependence of the $E_{0}$ gaps of these materials. The behavior of $E_{0}(T)$ is particularly interesting for $\mathrm{CuI}$ where we have nearly complete cancellation of the contributions of the copper and iodine vibrations to the temperature shift of $E_{0}$. For $\mathrm{CuBr}$, a reversal of the phonon eigenvectors was needed to explain the isotope effects, in agreement with results previously reported from Raman measurements.26

We have examined similar effects observed for the copper and silver chalcopyrites (I-III-VI 2 ) and pointed out that the contribution of the nobel metals is also negative but less than that in the binary halides. This follows naturally from the amount of the noble metal present in these compounds.

\section{ACKNOWLEDGMENTS}

J. S. acknowledges financial support from the MaxPlanck-Gesellschaft and the Ministerio de Educación, Cultura y Deportes (Spain) through the Plan Nacional de Formación del Profesorado Universitario.

${ }^{1}$ M. Cardona, Phys. Rev. 129, 69 (1963).

${ }^{2}$ A. Goldmann, Phys. Status Solidi B 81, 9 (1977).

${ }^{3}$ K. Shindo, A. Morita, and K. Kamimura, Proc. Phys. Soc. Jpn. 20, 2054 (1965).

${ }^{4}$ S. Ves, D. Glötzel, M. Cardona, and H. Overhof, Phys. Rev. B 24, 3073 (1981).

${ }^{5}$ A. Blacha, S. Ves, and M. Cardona, Phys. Rev. B 27, 6346 (1983).

${ }^{6}$ K. Reimann and St. Rübenacke, Phys. Rev. B 49, 11021 (1994).

7 A. Göbel, T. Ruf, M. Cardona, C. T. Lin, J. Wrzesinski, M. Steube, K. Reimann, J.-C. Merle, and M. Joucla, Phys. Rev. B 57, 15183 (1998).

${ }^{8}$ P. Etchegoin, J. Weber, M. Cardona, W. L. Hansen, K. M. Itoh, and E. E. Haller, Solid State Commun. 83, 843 (1992).

${ }^{9}$ C. Parks, A. K. Ramdas, S. Rodríguez, K. M. Itoh, and E. E. Haller, Phys. Rev. B 49, 14244 (1994).

10 M. Cardona, Phys. Status Solidi A, in press.

${ }^{11}$ L. F. Lastras-Martínez, T. Ruf, M. Konuma, M. Cardona, and D. E. Aspnes, Phys. Rev. B 61, 12946 (2000).

12 A. Göbel, T. Ruf, J. M. Zhang, R. Lauck, and M. Cardona, Phys. Rev. B 59, 2749 (1999).

${ }^{13}$ F. Raga, R. Kleim, A. Mysyrowicz, J. B. Grun, and S. Nikitine, J. Phys. (France) Colloq. 28, C3-116 (1967).

${ }^{14}$ Y. Kaifu and T. Komatsu, Phys. Status Solidi B 48, K125 (1971).

${ }^{15}$ V. K. Miloslavskii and O. N. Yunakova, Opt. Spectrosc. 57, 51 (1984).

${ }^{16}$ S. Lewonczuk, J. G. Gross and J. Ringeisen, J. Phys. (France) Lett. 42, L91 (1981).

17 S. Chichibu, H. Nakanishi, and S. Shirakata, Appl. Phys. Lett. 66, 3513 (1995).

18 M. Quintero, C. Rincón, R. Tovar, and J. C. Woolley, J. Phys.: Condens. Matter 4, 1281 (1992).

19 S.-H. Wei, A. Zunger, I.-H. Choi, and P. Y. Yu, Phys. Rev. B 58, R1710 (1998).

${ }^{20}$ A. Rivero, M. Quintero, Ch. Power, J. González, R. Tovar, and J. Ruiz, J. Electron. Mat. 26, 1428 (1997).

${ }^{21}$ M. Quintero, B. D. Marks, and J. C. Woolley, J. Appl. Phys. 66, 2402 (1989).

${ }^{22}$ L. Artus and Y. Bertrand, Solid State Commun. 61, 733 (1987).

${ }^{23}$ V. A. Aliyev, G. D. Guseinov, F. I. Mamedov, and L. M. Chapanova, Solid State Commun 59, 745 (1986).

${ }^{24}$ M. Quintero, R. Tovar, C. Bellabarba, and J. C. Woolley, Phys. Status Solidi B 162, 517 (1990). 
${ }^{25}$ S. Suga, K. Cho, and M. Bettini, Phys. Rev. B 13, 943 (1976).

${ }^{26}$ J. Serrano, T. Ruf, F. Widulle, C. T. Lin, and M. Cardona, Phys. Rev. B 64, 045201 (2001).

${ }^{27}$ C. T. Lin, E. Schönherr, A. Schmeding, T. Ruf, A. Göbel, and M. Cardona, J. Crystal Growth 167, 612 (1996).

${ }^{28}$ C. T. Lin et al., unpublished.

29 D. Fröhlich, in Nonlinear Spectroscopy of Solids: Advances and Applications, edited by B. Di Bartolo and B. Bowlby (Plenum, New York, 1994), p. 289.

30 D. Fröhlich, E. Mohler, and P. Wiesner, Phys. Rev. Lett. 26, 554 (1971). D. Frölich and W. Nieswand, Phil. Mag. B 70, 321 (1994).

${ }^{31}$ S. Suga, K. Cho, Y. Niji, J. C. Merle, and T. Sauder, Phys.
Rev. B 22, 4931 (1980).

${ }^{32}$ Y. Nozue, J. Phys. Soc. Jpn. 51, 1840 (1982).

${ }^{33}$ Landolt-Börnstein, Numerical Data and Functional Relationships in Science and Technology, Group III, Vols. 17a, 22a, and 41b (Springer-Verlag, Berlin, Heidelberg, 1982, 1999).

${ }^{34}$ J. Serrano, M. Cardona, and T. Ruf, Solid State Commun. 113, 411 (2000).

${ }^{35}$ M. Cardona, T. Ruf, and J. Serrano, Phys. Rev. Lett. 86, 3923 (2001).

36 T. Goto and T. Takahashi, J. Phys. Soc. Jpn. 24, 314 (1968).

${ }^{37}$ We have taken for this calculation $\alpha=-1.2 \mathrm{eV}(-1.10(7)$ from Ref. 33i). 
TABLE I. Dependence of the exciton energies on isotope mass and corresponding zero-temperature renormalizations. The values obtained by varying the isotopes of copper and halogen $(\mathrm{X})$ are compared with those found from two-oscillator fits to the temperature dependence, the latter labeled $T, L$ or $M$ according to the frequencies chosen for each oscillator, i.e., either TA and TO, or LA and LO, or a mixture of tranverse and longitudinal phonons of frequencies in the one-third to two-thirds ratio, respectively. In the case of $\mathrm{CuI}$, a three-oscillator model was necessary to fit the experimental data; we used the average frequencies of the TA, LA, and LO bands. The frequencies are given in $\mathrm{K}$, whereas the derivatives are displayed in $\mu \mathrm{eV} / \mathrm{amu}$. $E_{0}$ is given in meV.

\begin{tabular}{|c|c|c|c|c|c|c|c|c|c|c|c|}
\hline \multirow[b]{2}{*}{$\mathrm{CuX}$} & \multicolumn{4}{|c|}{ Isotope measurements } & \multicolumn{7}{|c|}{ Temperature data } \\
\hline & $\frac{\partial E_{0}^{\Gamma_{3}}}{\partial M_{\mathrm{Cu}}}$ & $\frac{\partial E_{0}^{\Gamma_{3}}}{\partial M_{\mathrm{X}}}$ & $\frac{\partial E_{0}^{\Gamma_{5}}}{\partial M_{\mathrm{Cu}}}$ & $\frac{\partial E_{0}^{\Gamma_{5}}}{\partial M_{\mathrm{X}}}$ & $\begin{array}{l}E_{0} \\
E_{0} \\
E_{0}\end{array}$ & $\begin{array}{c}\frac{\partial E_{0}^{T}}{\partial M_{\mathrm{Cu}}} \\
\frac{\partial E_{0}^{L}}{\partial M_{\mathrm{Cu}}} \\
\frac{\partial E_{0}^{M}}{\partial M_{\mathrm{Cu}}} \\
\end{array}$ & $\begin{array}{l}\frac{\partial E_{0}^{T}}{\partial M_{\mathrm{X}}} \\
\frac{\partial E_{0}^{L}}{\partial M_{\mathrm{X}}} \\
\frac{\partial E_{0}^{M}}{\partial M_{\mathrm{X}}} \\
\end{array}$ & $\omega_{\mathrm{TA}}$ & $\omega_{\mathrm{LA}}$ & $\omega_{\mathrm{TO}}$ & $\omega_{\mathrm{LO}}$ \\
\hline $\mathrm{CuCl}$ & $-81^{a}$ & $346^{a}$ & -71 & 382 & $\begin{array}{l}3236 \\
3260 \\
3244\end{array}$ & $\begin{array}{r}-69 \\
-446 \\
-152\end{array}$ & $\begin{array}{r}546 \\
1482 \\
773\end{array}$ & 51 & 169 & 300 & 333 \\
\hline $\mathrm{CuBr}$ & & & $-115(90)$ & $132(40)$ & $\begin{array}{l}2975 \\
2984 \\
2978\end{array}$ & $\begin{array}{r}-44 \\
-378 \\
-98\end{array}$ & $\begin{array}{r}87 \\
401 \\
142 \\
\end{array}$ & 53 & 158 & 216 & 233 \\
\hline$\overline{\mathrm{CuI}}$ & $-550(12)$ & & $-510(150)$ & & 3045 & -524 & 243 & 60 & 156 & 192 & 224 \\
\hline
\end{tabular}

${ }^{a}$ Because of the different band edge structure of $\mathrm{CuCl}$, these data do not correspond to a $\Gamma_{3}$ exciton but to the upper polariton. See Fig. 1 of Ref. 7 . 
TABLE II. Temperature shift of the lower absorption gap, $E_{0}$, for cuprous and silver chalcopyrites. The data are compared with that of the corresponding isoelectronic binary compounds, the cuprous halides, and other binary compounds with zinc-blende crystal structure. $T_{a}$ stands for the temperature at which a kink is observed in the temperature dependence, and the hydrostatic deformation potential is listed under $\alpha$. $E_{0}$ and $E_{0}(300 K)-E_{0}(0)$ are given in $\mathrm{meV}, \alpha$ in $\mathrm{eV}$, and $T_{a}$ in $\mathrm{K}$.

\begin{tabular}{|c|c|c|c|c|}
\hline & $E_{0}(T=0)$ & $E_{0}(300 K)-E_{0}(0)$ & $T_{a}$ & $\alpha$ \\
\hline${ }^{a} \mathrm{CuAlS}_{2}$ & 3510 & -45 & 140 & \\
\hline${ }^{b} \mathrm{CuGaS}_{2}$ & 2510 & -60 & 120 & \\
\hline${ }^{b} \mathrm{CuGaSe}_{2}$ & 1720 & -35 & 140 & $-3.9^{c}$ \\
\hline${ }^{d} \mathrm{CuGaTe}_{2}$ & 1444 & -88 & & $-4.6^{c}$ \\
\hline${ }^{b} \mathrm{CuInS}{ }_{2}$ & 1534 & -13 & 120 & \\
\hline${ }^{b} \mathrm{CuInSe}{ }_{2}$ & 990 & -23 & 100 & $-2.2^{c}$ \\
\hline${ }^{e} \mathrm{CuInTe}{ }_{2}$ & 1000 & -43 & 200 & $-3.4^{c}$ \\
\hline${ }^{f} \mathrm{AgGaS}_{2}$ & 2710 & -33 & 110 & \\
\hline${ }^{f} \mathrm{AgGaSe}_{2}$ & 1820 & -22 & 170 & $-3.2^{c}$ \\
\hline${ }^{g} \mathrm{AgInSe}_{2}$ & 1200 & +10 & 120 & $-1.7^{c}$ \\
\hline${ }^{h} \mathrm{AgInTe}_{2}$ & 1000 & -36 & & \\
\hline${ }^{i} \mathrm{CuCl}$ & 3206 & +50 & 100 & -0.4 \\
\hline${ }^{i} \mathrm{CuBr}$ & 2967 & +32 & 100 & -0.3 \\
\hline${ }^{i} \mathrm{CuI}$ & 3057 & -8 & 180 & -1.1 \\
\hline${ }^{i} \mathrm{AgI}$ & 2900 & -12 & & \\
\hline${ }^{i} \mathrm{GaAs}$ & 1540 & -95 & & -9.8 \\
\hline${ }^{i} \mathrm{GaSb}$ & 813 & -88 & & -7.0 \\
\hline${ }^{i} \mathrm{ZnSe}$ & 2770 & -90 & & -4.9 \\
\hline${ }^{i} \mathrm{ZnTe}$ & 2381 & -111 & & -5.9 \\
\hline
\end{tabular}

${ }^{a}$ Ref. 17

${ }^{b}$ Ref. 18

${ }^{c}$ Ref. 19

${ }^{d}$ Ref. 20

${ }^{e}$ Ref. 21

${ }^{f}$ Ref. 22

${ }^{g}$ Ref. 23

${ }^{h}$ Ref. 24

${ }^{i}$ Ref. 33

FIG. 1. Two-photon-absorption spectra of isotopically modified CuI measured at $7 \mathrm{~K}$. The two features observed in each spectrum correspond to the $\Gamma_{3}$ and the $\Gamma_{5}$ excitons. The dashed lines are guides to the eye. Note the anomalous negative shift in the peak energies with increasing copper mass.

FIG. 2. Isotope mass dependence of the $\Gamma_{3}$ and $\Gamma_{5}$ excitons in CuI. The lines represent least-squares fits to the experimental data from Fig. 1 .

FIG. 3. Temperature dependence of the lowest exciton peak for CuI (equivalent to that of the $E_{0}$ gap). The dotted line represents a three-oscillator fit of the difference between the experimental data (solid circles) and the contribution due to the thermal expansion (open diamonds), which is displayed shifted by $E_{0}(T=0)$ in order to show more clearly the relevance of this term. The dashed line is obtained by adding both contributions. The arrows indicate the frequencies of the three oscillators used in the fit (also listed in Tab. I). A small sigmoidal dependence is observed at low temperatures (see inset). Note the flattening of the temperature dependence at $\approx 180 \mathrm{~K}$.

FIG. 4. Two-photon-absorption spectra of $\mathrm{CuBr}$ for several isotopic compositions measured at $7 \mathrm{~K}$. The dashed lines are guides to the eye. An anomalous decrease of the exciton energy is observed with increasing copper mass (upper figure), whereas the lower figure displays the usual positive slope with increasing bromine mass. 
FIG. 5. Dependence of the low temperature exciton energy in $\mathrm{CuBr}$ on copper and bromine masses. The solid lines represent least-squares fits to the data of Fig. 4 


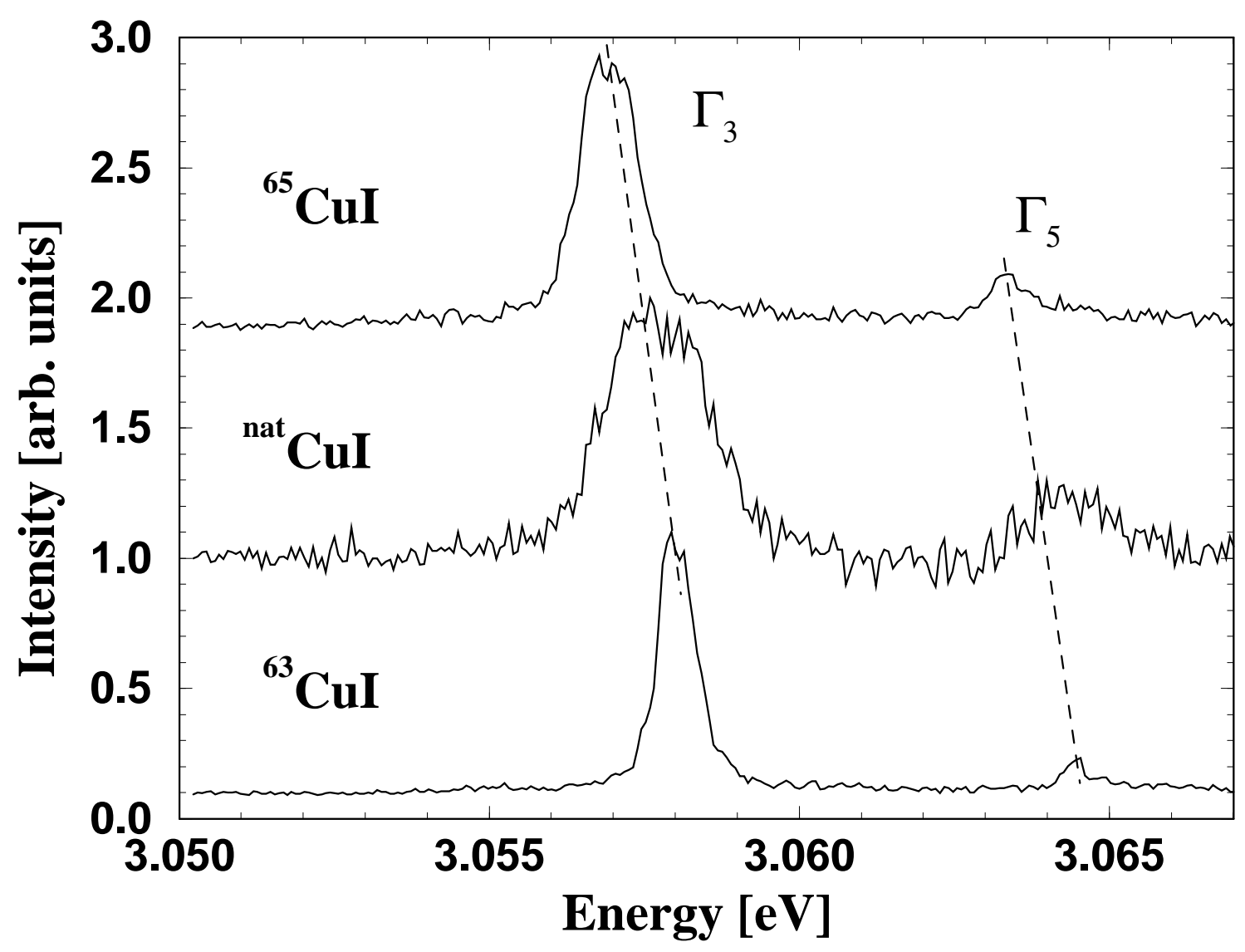

FIG. 1: J. Serrano et al. 

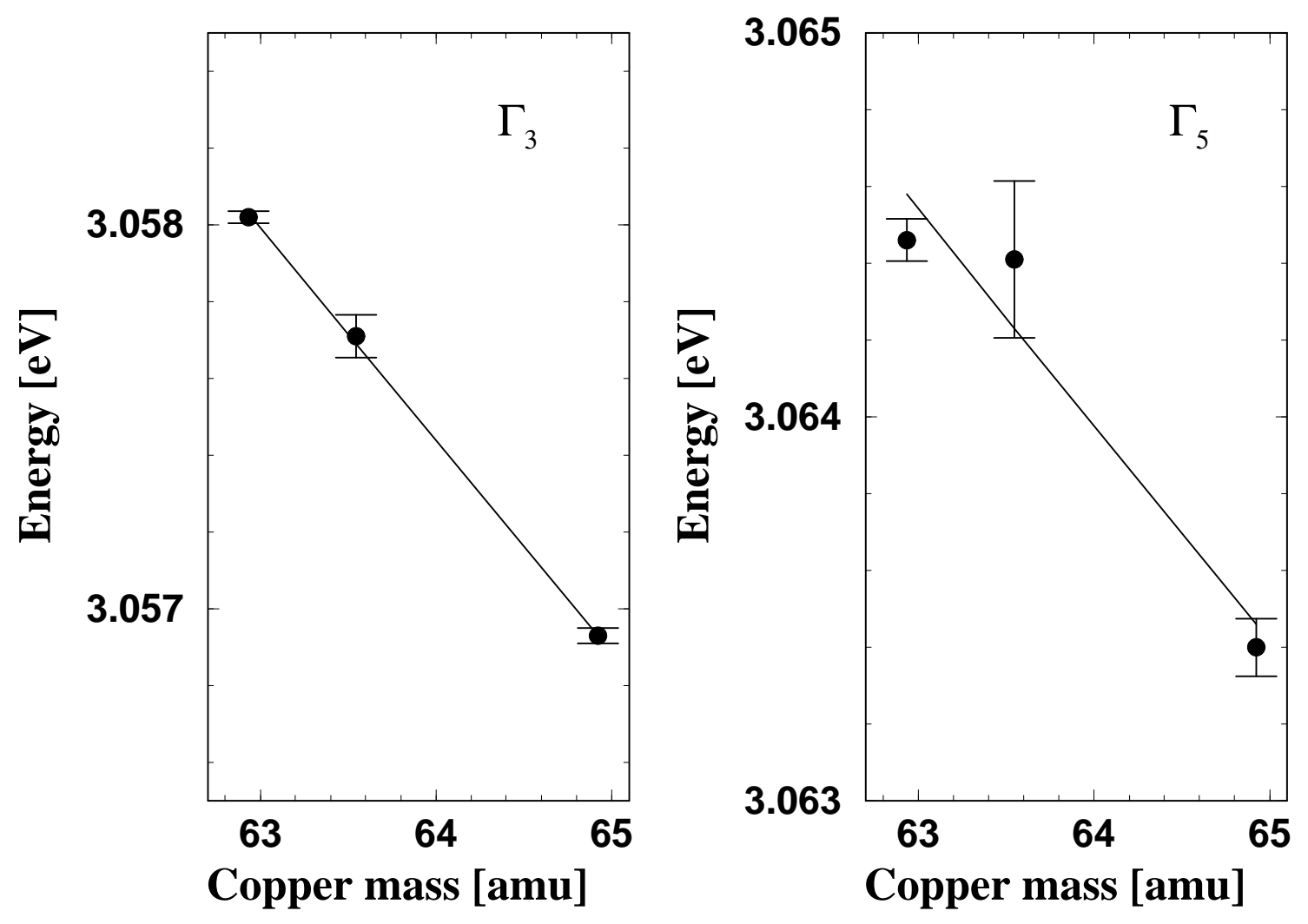

FIG. 2: J. Serrano et al. 


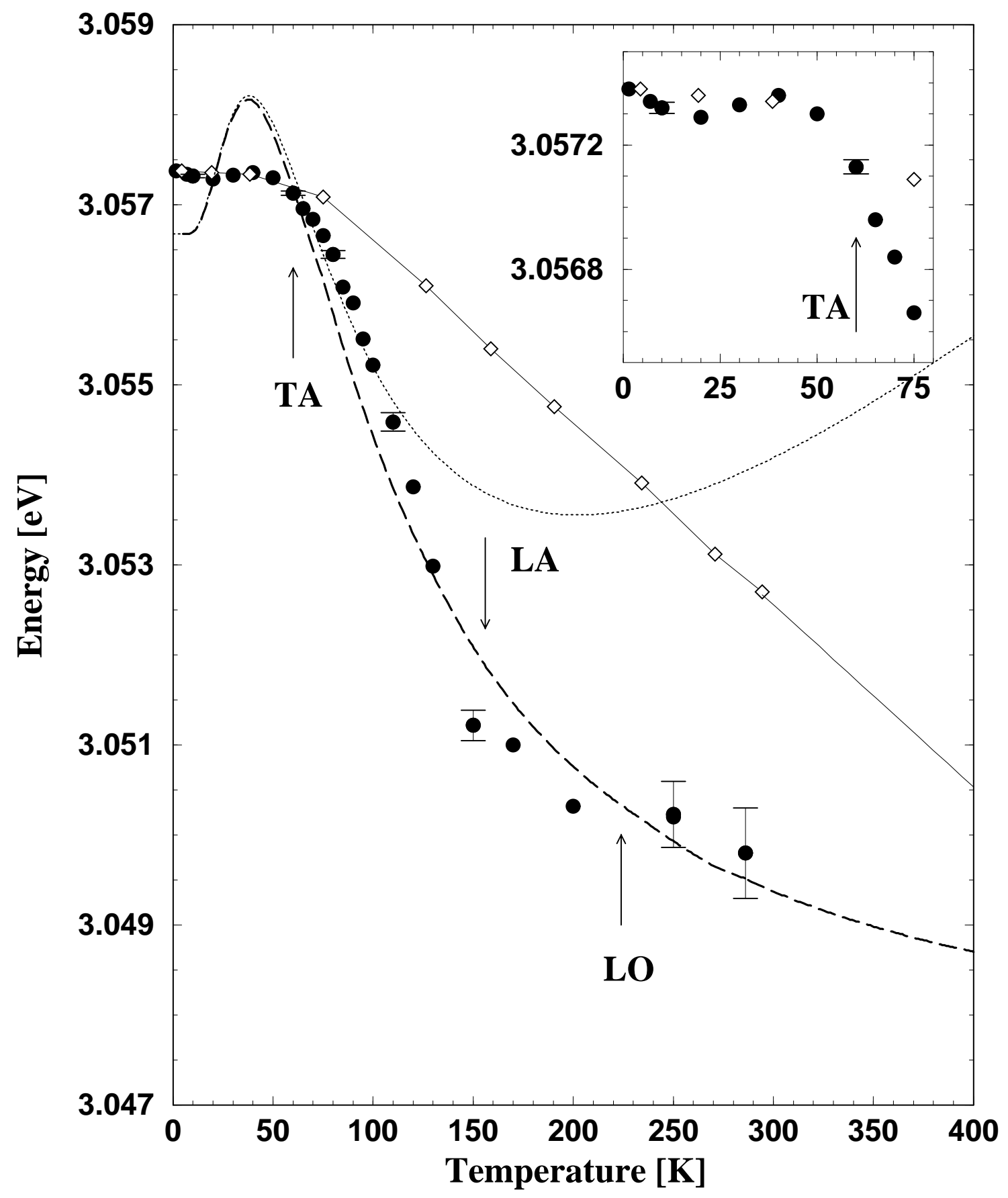

FIG. 3: J. Serrano et al. 

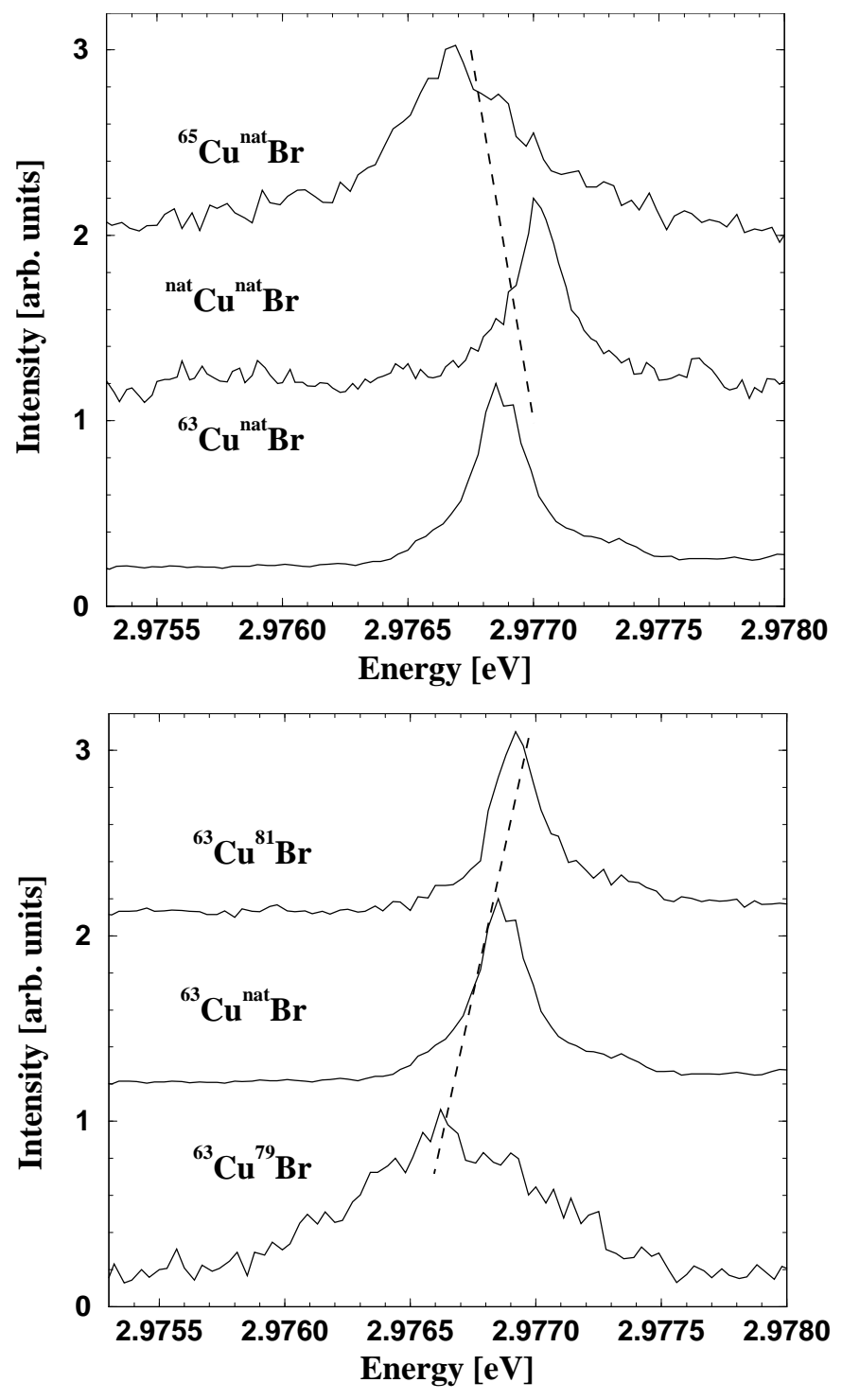

FIG. 4: J. Serrano et al. 

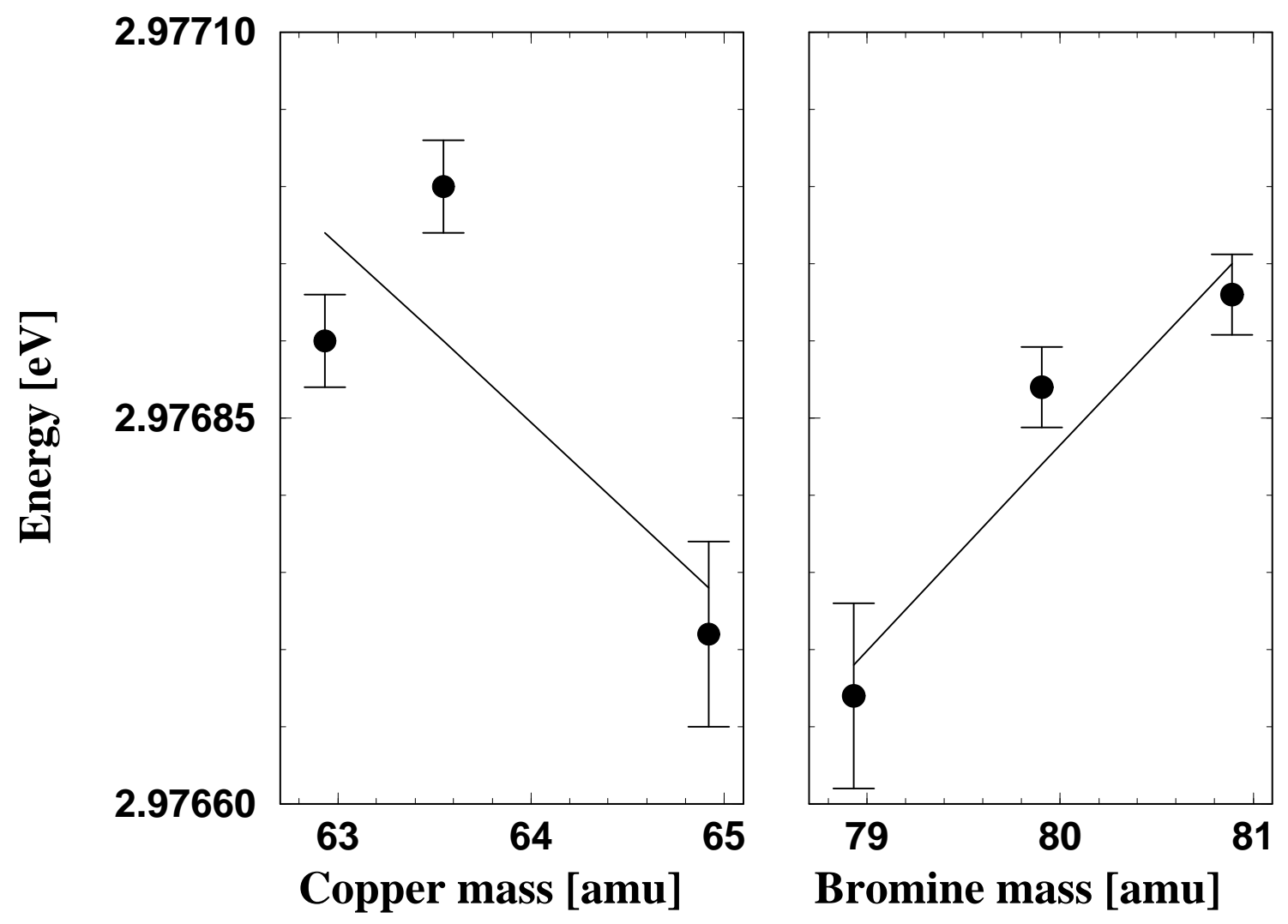

FIG. 5: J. Serrano et al. 